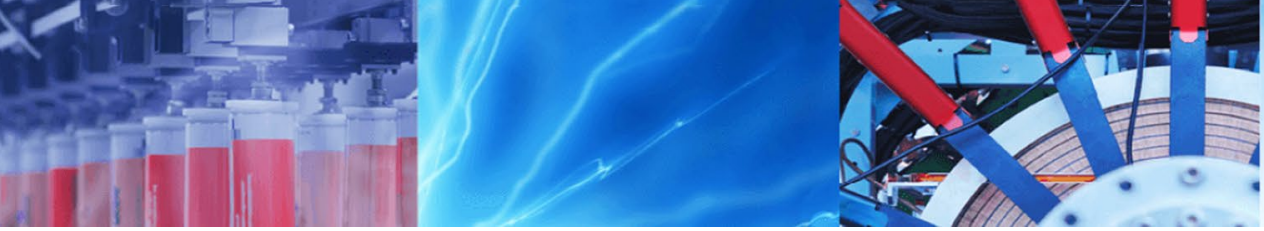

Research Article

\title{
On the use of fast Fourier transform for optical layer thickness determination
}

\author{
Michael Quinten $^{1}$ (D) \\ () Springer Nature Switzerland AG 2019
}

\begin{abstract}
Thin film thickness determination with a reflectometer is a fast and pretty cheap method that can be applied on many thin and thick films that are transparent or semitransparent in the considered spectral range. For evaluation of the reflectance spectrum either nonlinear regression analysis is used for very thin films or fast Fourier transform (FFT) for films thicker than approximately $1 \mu \mathrm{m}$. Using FFT for layer thickness determination there are some special facts to consider in contrast to the common use of FFT in signal processing. First of all, the sampling points are in general not equidistant as the wavelengths of the used spectrometer are not equidistant. Next the number of sampling points may be different from a power of 2 . The reason is that the measured spectral range may be restricted by the user. And finally the analogues in layer thickness determination to the independent parameters "time" and "frequency" in signal processing are not independent of each other. The reason is the dispersion of the refractive index of the layer material that causes unwanted Moire effects in the reflectance spectrum. All these deviations lead to additional sources for errors in the thickness determination beyond those error sources that are well-known from common FFT applications in signal processing. In this paper we discuss how these deviations affect the thickness determination and present a solution for the main problem caused by the dispersion of the refractive index. Further improvements of the FFT result in combination with a grid search algorithm and a nonlinear regression are presented and discussed. Finally we present with structured samples and highly doped semiconductors two specific applications of FFT on layer thickness determination.
\end{abstract}

Keywords Thin films · Layer thickness · FFT · Reflectometry · Patterned film · Highly doped silicon

\section{Mathematics Subject Classification $65 T 50$}

JEL Classification $\mathrm{C02} \cdot \mathrm{C} 63$

\section{Introduction}

Optical thickness determination with a reflectometer is a fast contactless method to control the thickness of one or more thin films in a layer stack. The film thickness is not directly obtained from the measurement but follows from mathematical algorithms applied on the measurement. For thin films below approximately 1-2 $\mu \mathrm{m}$ a nonlinear regression analysis is commonly used that allows for thickness evaluation even with nanometer resolution. For thicker layers the simpler and faster fast Fourier transform (FFT) can be applied. However, the application of FFT on layer thickness determination is restricted and needs improvements to obtain higher accuracy. Theoretically the FFT is exact and describes a function $f(t)$ which is periodical in time by an equivalent function $F(\omega)$ in the frequency domain. In principle it is applicable to all problems where a periodical function in real space ("time"-domain) shall be represented in the reciprocal space ("frequency"-domain). Actually, in most

Michael Quinten, michael.quinten@online.de| ${ }^{1}$ Wissenschaftlich-technische Software, Aldenhoven, Germany.

SN Applied Sciences (2019) 1:823 | https://doi.org/10.1007/s42452-019-0866-9 
applications discrete sampling points in a finite sampling interval in real space cause problems in the reciprocal Fourier space where also only discrete frequencies are obtained. The main problems are leakage and aliasing. There exist well-known methods to reduce leakage, e.g. the use of window functions, but it cannot be avoided completely as the sampling interval is always finite.

Beyond these well-known difficulties in the Fourier transform of discrete sampling points further problems arise in the application of the FFT on thickness determination of thin layers. They are specific for the layer thickness determination and need an extra analysis and treatment. The purpose of this paper is to introduce in these specific problems and to give proposals how to minimize the error in the thickness determination when using FFT. For this we first take a look on the optical thickness determination with a reflectometer. Then, we briefly review the FFT and point to the differences compared to the usual application in signal processing. We show that the dispersion of the refractive index of the layer material strongly influences the thickness determination with FFT. Particularly for large layer thickness this dispersion leads to undesired Moiré effects in the reflectance spectrum that render the thickness determination from the power spectrum more difficult. The effects can actually be observed in real measurements but could not yet be explained. We give an explanation and a solution for this problem by introducing a mathematical method that allows thickness determination up to almost the Nyquist frequency. We also propose methods to increase the accuracy of the FFT by combining it with a grid search algorithm and a nonlinear regression. In the last part we apply FFT directly on certain sample configurations, namely structured samples and highly doped semiconductors.

\section{Optical thickness determination with reflectometry}

Optical thickness determination is mostly carried out as reflectance measurement. The reason is that often the substrate on which a layer or a layer stack is deposited is opaque in the considered spectral range. The light reflected by the layer or even a layer stack gets collected by a spectrometer where it is spectrally resolved. A computer records the data, stores and displays them. Here also the mathematical analysis of the measured spectrum is carried out. For a more detailed deduction and discussion we refer for example to Quinten [1]. In this paper we restrict on the simplest case of one layer on a substrate (see Fig. 1).

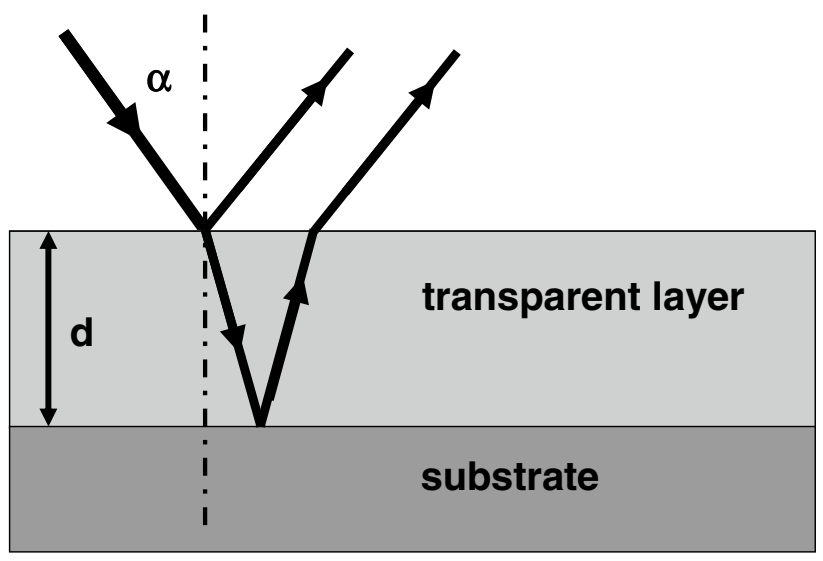

Fig. 1 Reflection at a transparent layer on a substrate

Considering all reflected and transmitted beams and all multiple beam interferences the reflectance spectrum of the layer is given by

$R(\lambda, d)=\frac{R_{01}(\lambda)+R_{12}(\lambda)+2 \sqrt{R_{01}(\lambda) \cdot R_{12}(\lambda)} \cdot \cos \left(\frac{4 \pi}{\lambda} n(\lambda) \cdot d\right)}{1+R_{01}(\lambda) \cdot R_{12}(\lambda)+2 \sqrt{R_{01}(\lambda) \cdot R_{12}(\lambda)} \cdot \cos \left(\frac{4 \pi}{\lambda} n(\lambda) \cdot d\right)}$

where $R_{01}$ is the reflectivity of the interface air-layer and $R_{12}$ is the reflectivity of the interface layer-substrate. The thickness of the film is $d$ and its refractive index is $n$. The measurement is usually carried out under normal incidence, i.e. the angle of incidence is $a=0^{\circ}$. Note that for $a \neq 0^{\circ}$ not only the reflectivities become angle-dependent and polarization-dependent but also the light path through the layer becomes longer and angle-dependent.

When restricting on the interference of only the first two reflected beams as shown in Fig. 1 Eq. (1) simplifies to

$$
\begin{aligned}
R(\lambda, d)= & R_{01}(\lambda)+R_{12}(\lambda) \cdot\left(1-R_{01}^{2}(\lambda)\right) \\
& +2 \sqrt{R_{01}(\lambda) \cdot R_{12}(\lambda) \cdot\left(1-R_{01}^{2}(\lambda)\right)} \\
& \cdot \cos \left(\frac{4 \pi}{\lambda} n(\lambda) \cdot d\right) .
\end{aligned}
$$

The reflectance is obviously composed of a wavelength dependent underground and an oscillating part given by the cosine term. Only this cosine term contains the information on the layer thickness. The film thickness can be determined using either a nonlinear regression analysis (particularly for thin films) or a fast Fourier transform (FFT). For the FFT at least one oscillation from maximum to maximum reflectance value is required. This condition is fulfilled for $d \approx 1-2 \mu \mathrm{m}$ in the wavelength range from 200 to $1700 \mathrm{~nm}$. This thickness is a reasonable but not very distinct limit for the applicability of the FFT. As it depends upon the refractive index of the layer this limit may be 
even lower for large refractive indices. Anyhow, the actual spectral range also plays an important role for the result of the FFT and the achievable resolution.

\section{Layer thickness determination with FFT}

For application of the FFT on layer thickness determination we first introduce analogues to the time variable and the frequency variable in common applications of the FFT in signal processing. The reflection gets sampled at discrete wavelengths $\lambda$, but when we look at Eqs. (1) or (2) we can interpret the variable $t=1 / \lambda$ as "time" variable and the variable $\omega=n \cdot d$ as "frequency" variable. If we apply now the discrete Fourier transform on Eq. (2) (or even Eq. (1)) using periodic functions $\exp \left(i \omega_{m} t\right)$ and $\exp \left(-i \omega_{m} t\right)$ we obtain the layer thickness

$\mathrm{d}_{\mathrm{FFT}}=\frac{\mathrm{m}}{2 \cdot \mathrm{n} \cdot\left(\mathrm{t}_{\max }-\mathrm{t}_{\min }\right)}=\frac{\mathrm{m}}{2 \cdot \mathrm{n} \cdot\left(\frac{1}{\lambda_{\min }}-\frac{1}{\lambda_{\max }}\right)}$

as an integer multiple $m$ of the minimum thickness

$\mathrm{d}_{\min }=\frac{1}{2 \cdot \mathrm{n} \cdot\left(\frac{1}{\lambda_{\min }}-\frac{1}{\lambda_{\max }}\right)}$.

If $N$ is the number of sampling points the Nyquist frequency respectively the maximum thickness that can be determined with FFT is

$\mathrm{d}_{\max }=\frac{\mathrm{N}-1}{2 \cdot \mathrm{n} \cdot\left(\frac{1}{\lambda_{\min }}-\frac{1}{\lambda_{\max }}\right)}=(\mathrm{N}-1) \cdot \mathrm{d}_{\min }$.

The boundaries $\lambda_{\min }$ and $\lambda_{\max }$ are given by the used spectrometer. The refractive index $n$ must be given by the user. Commonly, the wavelength range $\left[\lambda_{\min }, \lambda_{\max }\right]$ comprises $N$ nonequidistant wavelengths. Then, also the interval $\left[t_{\min } t_{\max }\right]=\left[1 / \lambda_{\min }, 1 / \lambda_{\max }\right]$ has $N$ nonequidistant values of $t$.

\section{Problems in optical layer thickness determination using FFT}

In signal processing a main source for errors is leakage. It is caused when the measured signal is not periodic to $100 \%$ in the sampling interval but exhibits discontinuities at the borders of the interval $\left[t_{\min }, t_{\max }\right]$. Then, the FFT spreads the power contained in the signal over all possible discrete frequencies $\omega_{k}$ with lower magnitudes of the modulus of the complex Fourier coefficients in the power spectral distribution (PSD). For example, if the smallest optical thickness according to Eq. (4) amounts to $(n \cdot d)_{\min }=8 \mu \mathrm{m}$ a periodic signal of a layer with optical thickness of $(n \cdot d)=100 \mu \mathrm{m}$ cannot be represented by one single discrete optical thickness that is an integer multiple of $8 \mu \mathrm{m}$ but gets spread over optical thicknesses $(n \cdot d)_{k}=k \cdot(n \cdot d)_{\min }$ meaning that $\cos (4 \pi / \lambda \cdot(n \cdot d))$ can only be represented by a sum over $\cos \left(4 \pi / \lambda \cdot k \cdot(n \cdot d)_{\min }\right)$ and $\sin \left(4 \pi / \lambda \cdot k \cdot(n \cdot d)_{\min }\right)$ terms, with $k=1, \ldots, N / 2$. This is illustrated in Fig. 2 .

This result is mathematically correct but not helpful in practice where the user wants to determine the actual thickness. The only possible opportunity is to search for the thickness with the biggest modulus in the PSD. In our example this is $96 \mu \mathrm{m}=12 \times 8 \mu \mathrm{m}$. It is fallacious to believe that the moduli obtained at the points $\mathrm{k} \cdot 8 \mu \mathrm{m}$ can be connected to a continuous curve (blue curve in Fig. 2) which can be fitted to find the best matching thickness. The thickness obtained by this way is not contained in the real reflectance spectrum. Moreover, its determination strongly depends on the current moduli of the Fourier coefficients. In our example the moduli imply that the best fit peak using a Lorentzian fit (magenta curve in Fig. 2) or a Gaussian fit (red curve in Fig. 2) lies at $92.5 \mu \mathrm{m}$ respectively $92.3 \mu \mathrm{m}$ which is close to $96 \mu \mathrm{m}$ but is farer away from $100 \mu \mathrm{m}$ than the peak with the biggest modulus. As a consequence of this consideration we must also state that the resolution in thickness determination with FFT is limited due to the discreteness of the sampling points, the finite interval of sampling points, and the selection made by the user and can amount to at best half the minimal evaluable thickness.

For the use of a fast Fourier transform some preconditions must be fulfilled. The first is that the FFT presumes

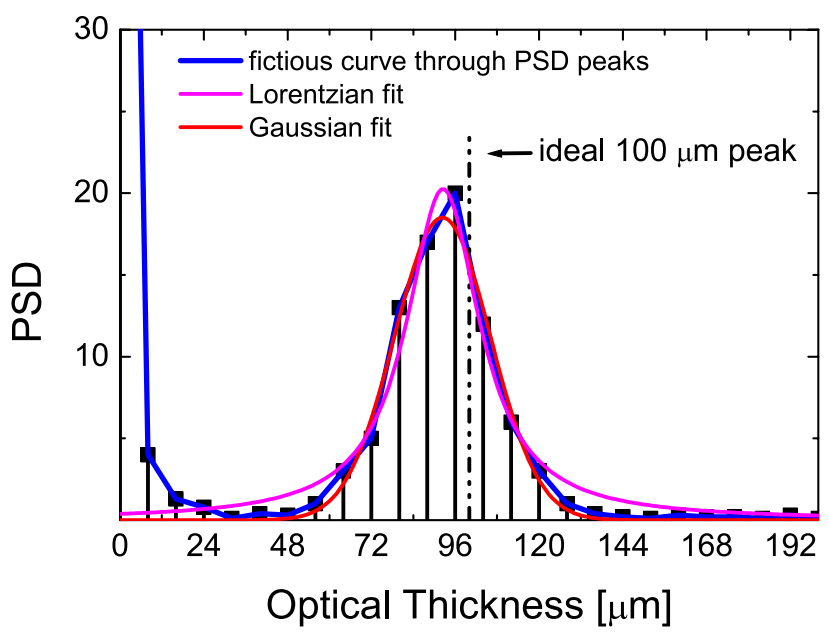

Fig. 2 Power spectral distribution (PSD) for a layer of optical thickness $100 \mu \mathrm{m}$ when the minimal evaluable thickness in FFT amounts to $8 \mu \mathrm{m}$ 
equidistant sampling points. This is critical in optical thickness determination since neither the wavelengths $\lambda$ of the used spectrometer nor the corresponding values of $t=1 / \lambda$ are equidistant if one uses a reflectometer with miniaturized spectrometer (diode line spectrometer). Then, the measured reflectance spectrum must be interpolated on $N$ equidistant sampling points with constant stepwidth $\Delta t=\left(t_{\max }-t_{\min }\right) /(N-1)=\left(1 / \lambda_{\min }-1 / \lambda_{\max }\right) /(N-1)$. The second condition is to have a number $N$ of sampling points that is either a power of 2 (radix 2 FFT) or a power of 4 (radix 4 FFT). This condition is not really problematic since there exist mixed radix algorithms that can treat also arbitrary numbers of sampling points. One advantage of a number $N$ matching this condition is that the algorithm needs less time. When using spectrometers with diode line detectors, $N$ is usually a power of 2 , i.e. 512,1024 , or 2048 pixels. In FTIR the number $N$ can differ from a power of 2. When looking at the measured reflectance spectrum in detail there are also reasons for what the evaluation of the spectrum may be restricted on a smaller wavelength range and hence on a smaller number $N$ of data points. The most prominent reason is that a part of the spectrum is very noisy caused by strong absorption in this wavelength range or by too low light from the used light source. As an example Fig. 3 shows the spectrum of a durimide photoresist layer on silicon (thickness approx. $12 \mu \mathrm{m}$ ) measured using a halogen light source and a miniaturized spectrometer with a spectral range of approximately $200-1050 \mathrm{~nm}$ and 2048 pixels. The absorption of light in durimide causes that the magnitude of the fringes decreases with decreasing wavelength. At wavelengths below approximately $520 \mathrm{~nm}$ the absorption is as high in this $12 \mu \mathrm{m}$ layer as the fringes have vanished and the spectrum becomes noisy. In this case it seems advantageous to use only the spectrum

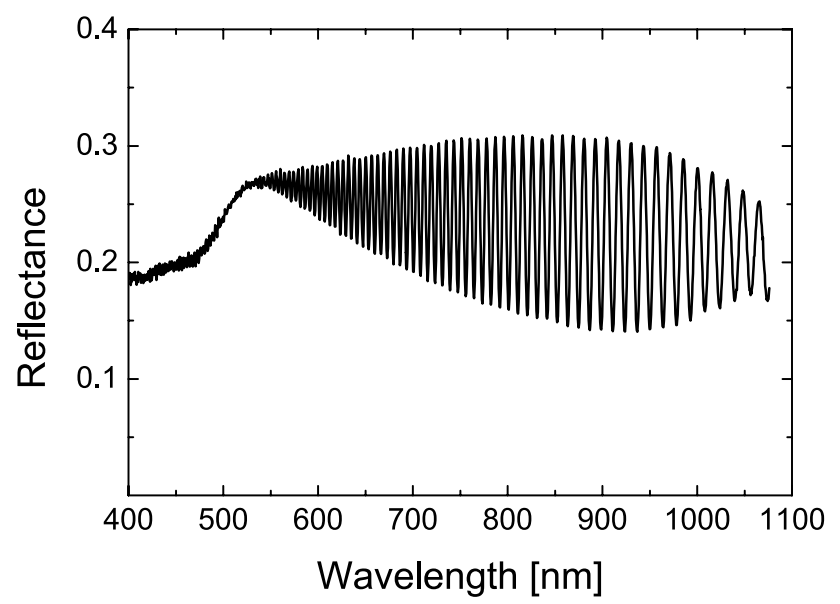

Fig. 3 Reflectance spectrum of a durimide film (approximately $12 \mu \mathrm{m}$ thickness) on a silicon substrate above approximately $550 \mathrm{~nm}$ for evaluation of the layer thickness.

The number of sampling points is then reduced to a number $N_{\text {eff }}$ of effective sampling points. This number may be not a power of 2 . As the reflectance data measured with a miniaturized spectrometer always need an interpolation on equidistant sampling points this can be combined with a number of points that is a power of 2. For example if $N_{\text {eff }}=734$ a number $N_{F F T}=1024$ can be chosen for the FFT. The interpolation of the 734 nonequidistant sampling points on 1024 equidistant points for the FFT means an oversampling. This oversampling however does not corrupt the result of the FFT but vice versa improves it. On the other hand the reduction of the number of sampling points means a reduction of the used spectral range. According to Eqs. (4)-(6) this affects the minimum and maximum evaluable thickness and hence the resolution of the FFT.

The main problems in optical layer thickness determination with FFT compared to its application in signal processing are however:

1) The refractive index $n$ is not constant in the wavelength range but depends upon the wavelength. This dispersion is missing in signal processing but is a problem here, since in effect it does not allow to separate the "time" (here: the wavenumber $t=1 / \lambda$ ) from the "frequency" (here: the optical thickness $\omega=n(1 / \lambda) \cdot d$ ). Moreover, the dispersion intensifies the second problem.

2) In miniaturized spectrometers the wavelengths and hence the variable $t=1 / \lambda$ has values with variable spacing, i.e. $t \neq t_{\min }+m \cdot \Delta t$. Rather, $\Delta t_{n}=t_{n+1}-t_{n}$ is varying. But also even if the spacing is constant as in FTIR spectrometers the dispersion of the refractive index acts similar to a variable spacing in $t$.

In the following, we discuss both problems and show how to improve the FFT analysis.

The use of a constant refractive index in Eq. (3) results in uncertainties in the thickness determination of up to $5 \%$ for highly refractive materials like semiconductors and up to $2 \%$ for other materials. The uncertainty can be reduced by using an effective refractive index $n_{\text {eff }}$ for the material under consideration. This effective refractive index is mostly remarkably larger than any actual refractive index of the material in the considered spectral range. For example, when investigating silicon diaphragms or even wafers the preferred wavelength range is the near infrared since silicon is transparent in this region. The actual refractive indices of silicon in this spectral range exhibit low dispersion and a value of around $n=3.51$ [2-4]. Using this value in 
Eq. (3) for evaluation of the reflectance of a $100 \mu \mathrm{m}$ thick silicon wafer in the spectral range from approximately $1260-1360 \mathrm{~nm}$ leads to a thickness of $d_{F F T}=105 \mu \mathrm{m}$ instead of actual $d=100 \mu \mathrm{m}$. The effective refractive index must be adjusted to $n_{\text {eff }}=3.69$ to obtain the correct thickness. This value for $\mathrm{n}_{\text {eff }}$ is however far beyond the actual refractive index values of silicon in this wavelength range.

Yet, it has been proven $[1,5,6]$ that the uncertainty can also be reduced down to less than $2 \%$ for high refractive materials and down to less than $1 \%$ for other materials when using

$d_{\min }=\frac{1}{2 \cdot\left(\frac{n\left(\lambda_{\min }\right)}{\lambda_{\min }}-\frac{\mathrm{n}\left(\lambda_{\max }\right)}{\lambda_{\max }}\right)}$.

as minimum thickness instead of Eq. (4). On the other hand, this formula is helpful to determine the effective refractive index $n_{\text {eff }}$ if $n\left(\lambda_{\min }\right)$ and $n\left(\lambda_{\max }\right)$ at the borders of the measuring interval are known:

$\mathrm{n}_{\text {eff }}=\frac{\left(\frac{\mathrm{n}\left(\lambda_{\min }\right)}{\lambda_{\min }}-\frac{\mathrm{n}\left(\lambda_{\max }\right)}{\lambda_{\max }}\right)}{\left(\frac{1}{\lambda_{\min }}-\frac{1}{\lambda_{\max }}\right)}$.

Both corrections Eqs. (6) and (7) may however fail if the dispersion of the refractive index becomes too strong. E.g., if one measures the thickness of a silicon wafer or diaphragm in the wavelength range around the interband transition at $1100 \mathrm{~nm}$ wavelength or at even smaller wavelengths, the dispersion becomes too strong to apply Eq. (6) or to use Eq. (3) with the effective refractive index from Eq. (7). Then, it seems appropriate to evaluate single fringes in the reflectance spectrum instead of applying FFT.

That means that the evaluation with FFT is replaced with an evaluation of the actual fringes in the reflectance spectrum. The thickness is obtained from two neighboring maxima to

$d=\frac{1}{2 \cdot\left(\frac{\mathrm{n}\left(\lambda_{1}\right)}{\lambda_{1}}-\frac{\mathrm{n}\left(\lambda_{2}\right)}{\lambda_{2}}\right)}$

where $\lambda_{1}$ and $\lambda_{2}$ are the wavelengths where the reflectance spectrum has maxima with $\lambda_{1}<\lambda_{2}$. This method should be applied on a small spectral range to improve the result by statistics. It presumes however the knowledge of the refractive indices of the layer material at the used wavelengths. There exist numerous works on the determination of optical constants with either tabulated data, graphical representation, or a parametrization of the data for a certain model. The Handbook of Optical Constants of Solids
[2], edited by Edward D. Palik, affords the most comprehensive database of the refractive index and absorption index of technically important and scientifically interesting dielectrics, semiconductors, and metals in three volumes. Another comprehensive sources of tabulated data are the Handbook of Optics II [7], the CRC Handbook of Chemistry and Physics [8], and particularly for semiconductors the book Optical Constants of Crystalline and Amorphous Semiconductors: Numerical Data and Graphical Information [9] by Sadao Adacho. A special reference for particularly thin films is the book The Optical Constants of Bulk Materials and Films by L. Ward [10]. It covers the theoretical background, experimental techniques, and results for a wide range of materials of thin films. Beyond that, optical constants are published in further books and in numerous articles in several journals. Many of the data in these references are also available from online databases like http://refractiveindex info and www.luxpop.com. Also some suppliers of measurement equipment provide databases, e.g. Filmetrics Inc. at http://www.filmetrics.com/refractive-index-database.

The second main problem with FFT in layer thickness determination arises from the fact that in the often used miniaturized spectrometers the wavelengths in the useful wavelength range gets projected on a diode line detector, causing that the discrete wavelengths are not equidistantly distributed over the pixel array. Then, also the parameter $t=1 / \lambda$ is not equidistant in the interval [ $t_{\text {min }}$ $t_{\text {max }}$. As already mentioned before, the measured reflectance spectrum must then be interpolated on $N$ equidistant sampling points $\Delta t=\left(t_{\max }-t_{\min }\right) /(N-1)=\left(1 / \lambda_{\min }-1 /\right.$ $\left.\lambda_{\text {max }}\right) /(N-1)$. Unfortunately, this transformation does not override the Moiré pattern caused by the non-equidistant spacing in the original wavelength-dependent reflectance spectrum. To demonstrate this Moiré pattern we calculated a simple cosine function $\cos (\omega \cdot \mathrm{t})$ in the interval $t=[75 \mu \mathrm{m}$, $87.775 \mu \mathrm{m}$ ] with (1) an equidistant spacing $\Delta t=0.025 \mu \mathrm{m}$ (512 values) and (2) with $\Delta t=0.02262 \mu \mathrm{m}$ in the first third of the interval, $\Delta t=0.024882 \mu \mathrm{m}$ in the middle third, and $\Delta t=0.02753 \mu \mathrm{m}$ in the last third (also 512 values) and a fixed frequency $\omega=16 \pi \mu \mathrm{m}^{-1}$. The results are shown in Fig. 4 where the cosine function for the non-equidistant spacing (red curve) is shifted along the ordinate by adding the constant value 2 for better presentation. Obviously, the non-equidistant spacing introduces periodic variations in the magnitude of the cosine function similar to a Moiré effect although we have used only three different spacings which each even being constant over a third of the complete interval. However the phase of the cosine function is rather large amounting to approximately 3800-4400. Then, the cosine function rapidly changes between maximum to minimum. That means that such Moiré patterns can be expected for a large phases of the corresponding 


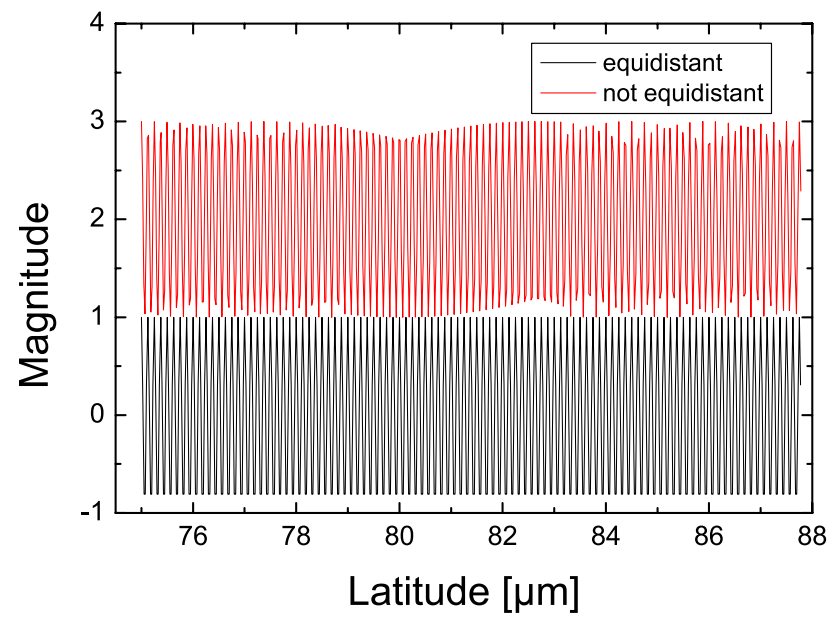

Fig. 4 The function $\cos (\omega \mathrm{t})$ calculated in the interval $t=[75 \mu \mathrm{m}$, $87.775 \mu \mathrm{m}]$ with fixed frequency $\omega=16 \pi \mu \mathrm{m}^{-1}$. The black curve is for an equidistant stepwidth $\Delta t$, for the red curve $\Delta t$ varies

periodic function. For small phase values the Moiré pattern is less obvious.

As however this Moiré pattern introduces periodic alterations of the signal, the introduced periods will be detected as additional frequencies in the power spectrum, disturbing the peak finding in the PSD. In practice, we could try to prevent non-equidistant spacing but with enormous effort. Unfortunately even if we would succeed in an equidistant spacing of $1 / \lambda$ we have to account for the dispersion $n(\lambda)$ of the refractive index of the layer material. This dispersion leads in any case to a non-equidistant spacing of the phase $4 \pi / \lambda \cdot n(\lambda) \cdot d$.

In the following we demonstrate in a series of pictures in Fig. 5 how the reflectance develops in the wavelength interval [1246 nm, $1373.75 \mathrm{~nm}$ ] and how the PSD and the thickness determination is affected by the frequencies introduced by the Moiré pattern. The calculations are carried out for an unsupported alumina layer with optical constants taken from [2] and interpolated on 512 equidistant wavelengths in the above interval. In each picture the reflectance spectrum is shown at left and the corresponding PSD at right. For better presentation of the PSD the peak at zero frequency is reduced and the PSD is normalized so that the maximum modulus of the Fourier coefficients amounts to 10 . For this wavelength interval the minimum thickness according to Eq. (4) for $n=1$ is $d_{\min }=6.699 \mu \mathrm{m}$, and the Nyquist frequency is $511 \cdot d_{\min }=3423 \mu \mathrm{m}$. In all pictures the size of the aluminum layer in the calculations was chosen to $d=m \cdot d_{\min } / n_{\text {eff }}$ with $n_{\text {eff }}=1.769$ for alumina in this spectral range. Then, the layer thickness frequency in the PSD is peaked at the FFT pixel $m$. In the pictures we therefore only give this number $m$ instead of the explicite thickness $d$.
Obviously the disturbing frequencies caused by the Moire pattern in the reflectance exhibit the following properties:

- They appear as a frequency band.

- This frequency band becomes noticeable above a certain layer thickness and appears at the high frequency end of the PSD while the layer thickness peak is still at low frequencies. In our example this band appears already for $m=100$.

- The frequency band shifts to lower frequencies with increasing layer thickness indicating that the contained frequencies are alias frequencies of frequencies higher than the Nyquist frequency. Moreover, the moduli of the corresponding Fourier coefficients increase when shifting from high to low frequencies.

As long as the modulus of the Fourier coefficient of the layer thickness frequency is clearly higher than the moduli of the coefficients of the alias frequencies a sophisticated peak search algorithm will always find the layer thickness frequency. This becomes increasingly more complicated if the alias frequency band is shifted to frequencies lower than the layer thickness frequency. The simultaneous increase of these moduli and decrease of the modulus of the layer thickness frequency makes it impossible to retrieve the layer thickness beyond a certain thickness. Unfortunately this limiting thickness is still far from the Nyquist frequency, in our example at $361 \cdot d_{\min }$. Improvement is achieved when using a quality factor $Q$ of the detected peak. Following the definition of the quality factor of a resonator, a definition of $Q$ can be the area under the peak within the full width at half maximum (FWHM) which is proportional to the product of peak height and FWHM. With the help of such a quality factor, the peak at the layer thickness frequency is found still beyond the above limiting thickness of $361 \cdot d_{\text {min }}$. Unfortunately this $Q$-factor only helps up to a thickness of $384 \cdot d_{\min }$ in our exemplaric calculations and with our realized calculation of $Q$. So, the Moiré pattern prevents in any case thickness determination above a certain thickness independent of how sophisticated the $Q$-factor is defined and calculated.

A possible solution of this problem is the following procedure. Instead of searching for a peak in the PSD we first multiply the PSD values with $m^{2}$ and then search for peaks in this modified PSD. This trick enormously reinforces the modulus of layer thickness peaks at high frequencies and simultaneously decreases drastically the moduli of the alias frequencies in comparison to the layer thickness peak. This procedure has been tested for PSDs of layers with thickness values $m=3 \cdot d_{\min }$ to $\mathrm{m}=511 \cdot d_{\min }$. For small thickness one can expect that the layer thickness peak still remains unaffected but the noise in the modified PSD will 
(a)

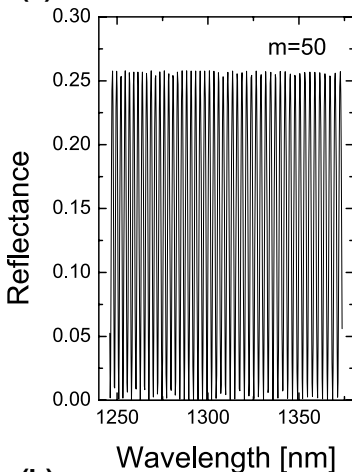

(b)

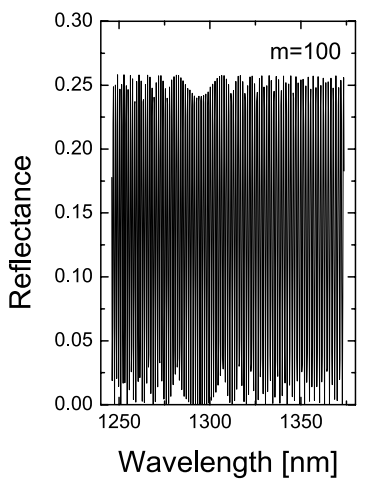

(c)

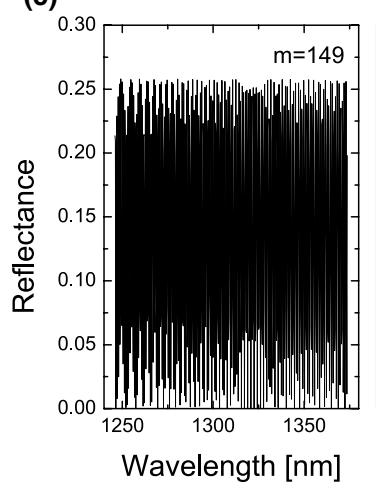

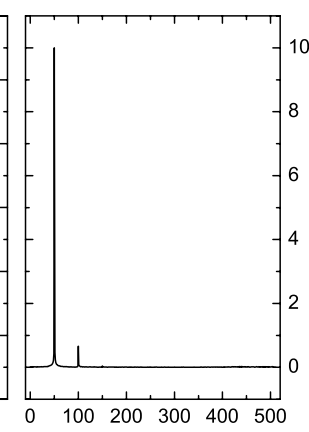

FFT Pixel No. m

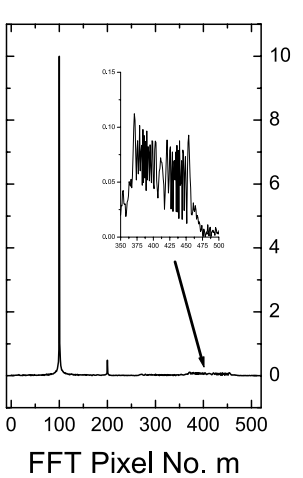

FFT Pixel No. $m$

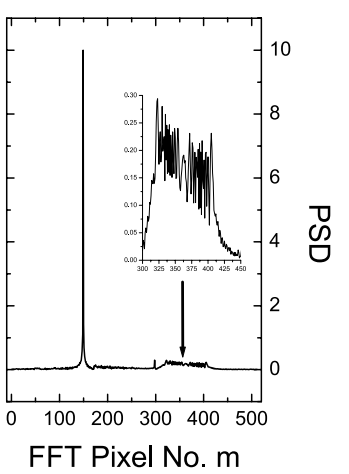

(g) 0.30

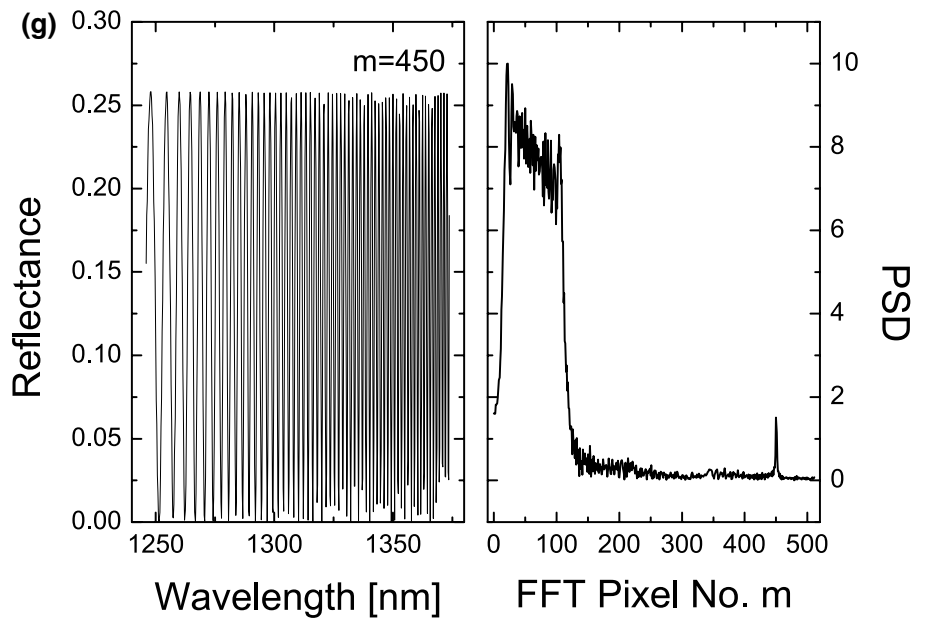

(d)

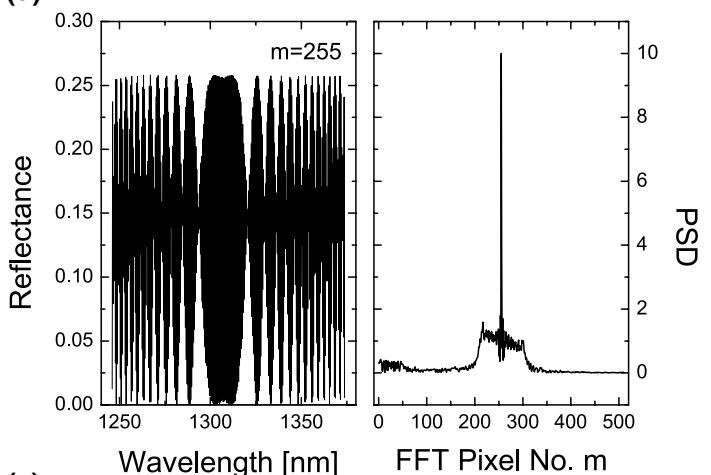

(e)

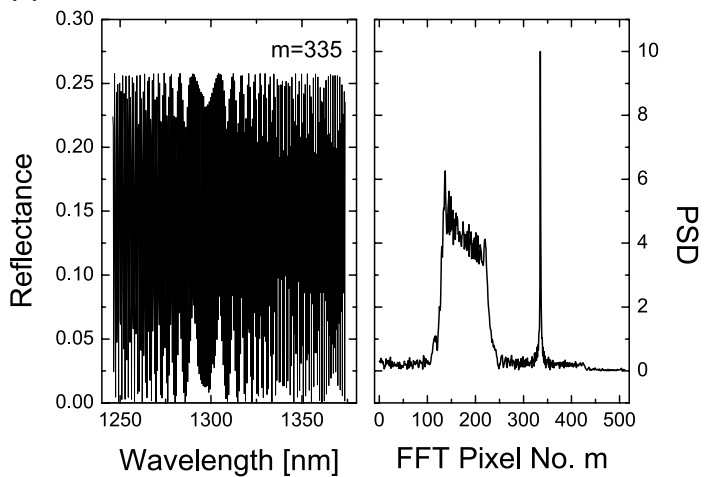

(f)



Fig. 5 Series of reflectance spectra and power spectral distributions for alumina layers of thickness $d=m \cdot d_{\min }$ 


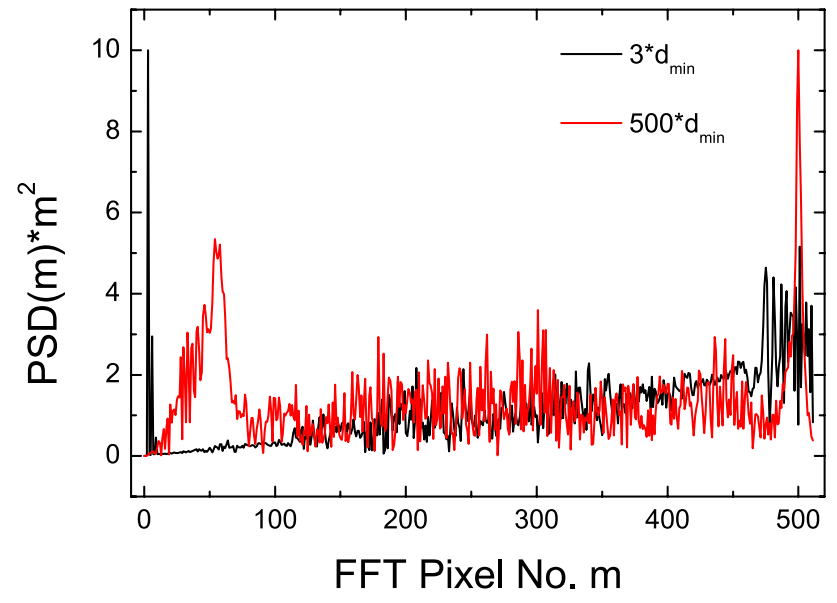

Fig. 6 Modified PSD for a layer with $d=3 \cdot d_{\min }$ and $d=500 \cdot d_{\min }$

increase at high frequencies. This can indeed be recognized in Fig. 6 for $m=3$ (black curve). On the other hand, for larger thickness we expect now a clearly resolved layer thickness peak which is significantly larger than all peaks in the alias frequency band. This is illustrated in Fig. 6 for $m=500$ (red curve). With this method we can retrieve a layer thickness up to $500 \cdot d_{\min }$. For still larger thickness up to $510 \cdot d_{\min }$ we observed that the weighting with $\mathrm{m}^{2}$ leads to a shift of the peak position of $-d_{\text {min }}$, e.g. instead of $d=505 \cdot d_{\min }$ we obtained $d=504 \cdot d_{\min }$. This method can reasonably applied if the minimal thickness $d_{\text {min }}$ according to Eq. (4) for $n=1$ is $d_{\min } \geq 4 \mu \mathrm{m}$. Then, a quality factor $Q$ is redundant.

Our investigations were not restricted on alumina layers but we carried out many further calculations with different materials that are transparent in the considered wavelength range. All calculations revealed the same behavior in reflectance and PSD for all tested materials so that we claim that our results can be applied on any transparent material for which the thickness shall be determined using Fourier transform of the reflectance spectrum.

\section{Improvements of the result of the FFT analysis}

As already mentioned the resolution in optical thickness determination with FFT is limited due to the discreteness of the sampling points, the finite interval of sampling points, and the selection made by the user. It amounts to at best half the minimal evaluable thickness. In the following we propose methods to improve the result of the FFT analysis. This can be achieved in two consecutive steps which however presume a possibility to calculate exactly the reflectance of the layer stack for a given layer thickness. If this model is available the first step is a grid search (a)

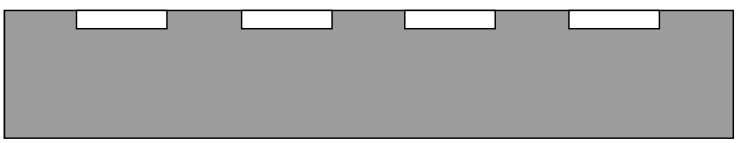

(b)

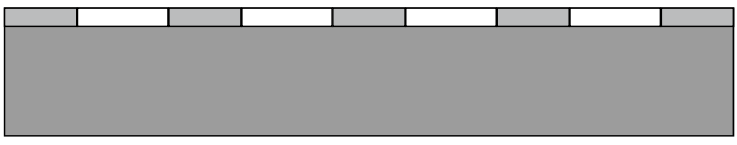

Fig. 7 a Transparent film on structured substrate. b Transparent film on substrate with a second film of another material on the same substrate

algorithm that calculates the reflectance in the interval $\left[d_{F F T}-2 \cdot d_{m i n}, d_{F F T}+2 \cdot d_{\min }\right]$ with $d_{F F T}$ being the result of the FFT analysis and $d_{\min }$ is the smallest evaluable thickness. It is convenient to divide this interval into an even number $M$ of points, e.g. $M=16$. Then, the results of this layer stack calculation are compared using the quadratic deviation $\chi^{2}$ of calculated and measured spectrum. The thickness for which this figure is the smallest already represents an improved result compared to $d_{F F T}$ with an accuracy of $4 \cdot d_{\min } / M$. Further improvement up to an accuracy of $0.1 \mathrm{~nm}$ can be obtained in a second step by using the thickness obtained from the grid search algorithm as initial value for a nonlinear regression with either the Levenberg-Marquardt algorithm [11, 12] or a downhill simplex algorithm according to Nelder and Mead [13]. The disadvantage of both steps is that they need time. However, on fast computers of today each single calculation is in the order of $10 \mathrm{~ms}$ or less. As the result of step 1 is already an initial value that lies close to the result of the nonlinear regression, the nonlinear regression often succeeds in the final result after about 20 iterations. So, for $M=16$ we have approximately an additional time and effort in the order of 400 ms but an increase in the accuracy of minimum a factor of 500-1000. This method seems therefore worth to think about.

\section{Specific applications of fast Fourier transform in layer thickness determination}

\subsection{Patterned film on substrate}

In some applications, e.g. in photolithography, a further treatment of the layer leads to structured surfaces where the original layer is reduced on smaller areas. Then, the optical thickness determination of the film is rendered more difficult because the reflection of the layer is superposed or mixed with the reflection of the substrate or a 
second layer of another material adjacent to the film under consideration as illustrated in Fig. 7.

Li and Lee [14] already gave a sophisticated calculation of the intensity reflected by a patterned film. We restrict on a simpler approach and examine when we get an unambigous signal from the patterned film that can be evaluated with FFT.

One possibility to get information on the thickness of the film is to use a microscope with a small field of view when measuring the reflectance of the film. This solution is expensive and suffers from remarkable loss of light intensity on the one hand and from a high sensitivity on the correct focus position. Moreover, a high numerical aperture of the microscope objective introduces also a dependence on the angle of incidence on the sample. This affects the size determination as demonstrated in $[5,6]$. Another approach is presented in this paper. In a numerical study on the thickness determination for structured samples we showed that it is possible to determine the film thickness exactly from the superposed signal

$R(\lambda)=x \cdot R_{\text {layer }}(\lambda)+(1-x) \cdot R_{\text {substrate }}(\lambda)$

when the portion $x$ as percentage of the layer area to the total area of the detection spot becomes larger than a threshold value $x_{t h}$. For this we investigated layers of $\mathrm{SiO}_{2}$, $\mathrm{Si}_{3} \mathrm{~N}_{4}$, and of the photoresist material durimide on substrates of $\mathrm{Si}$ or $\mathrm{GaAs}$ and varied the film thickness from $d=2 \mu \mathrm{m}$ to $d=20 \mu \mathrm{m}$ to determine the threshold value. To get more realistic results the computed reflectance spectra were additionally made noisy.

In Fig. 8a we exemplarily show the smooth spectra of a silica film of $d=5 \mu \mathrm{m}$ on a silicon substrate for $x=0.05$ and $x=0.10$ in comparison to the spectrum of the uncoated silicon substrate $(x=0)$, and in Fig. $8 \mathrm{~b}$ the corresponding power spectral distributions from FFT analysis where the blue curve is the PSD for $x=1.0$ (continuous film).

The PSDs exhibit a peak at a pixel number $m$ from which the thickness can be derived. The modulus of the Fourier coefficient at the peak position increases with increasing $x$ but for an unambiguous determination of the thickness a threshold value of $x_{t h}$ should be reached. This threshold value has been derived from our calculations and the results are summarized in the following Table 1. We can conclude that in all investigated cases a threshold value of $x_{t h}=0.1$ for smooth spectra and $x_{t h}=0.15$ for noisy spectra is sufficient to determine unambiguously the thickness of the film from the mixed reflectance spectrum.

\subsection{Interferometric thickness of highly doped semiconductors}

In the second application example we deal with thin wafers of semiconductors. Semiconductors are mostly
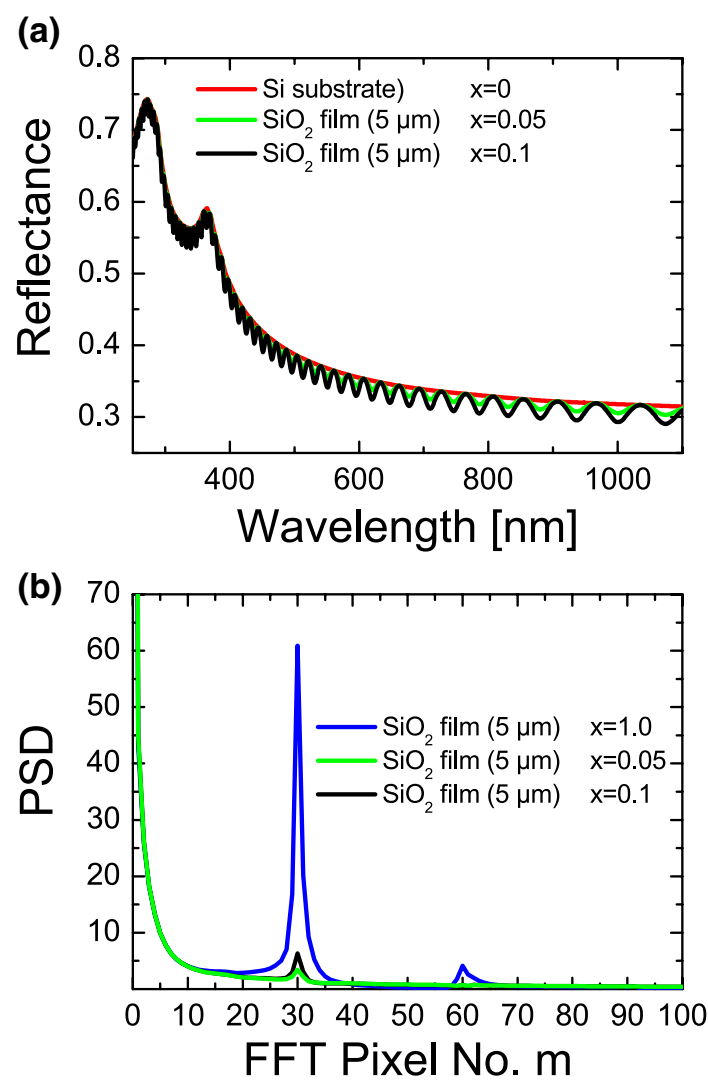

Fig. 8 a Exemplaric calculated spectra of a silica film on silicon substrate for $x=0$ (substrate only), $x=0.05$, and $x=0.1$, b Corresponding power spectral distributions in comparison to the PSD of the homogeneous film $(x=1.0)$

Table 1 Results for the threshold value

\begin{tabular}{llll}
\hline Film material & Substrate & Smooth spectra & Noisy spectra \\
\hline $\mathrm{Al}_{2} \mathrm{O}_{3}$ & $\mathrm{Al}$ & 0.05 & 0.08 \\
$\mathrm{SiO}_{2}$ & $\mathrm{Si}$ & 0.10 & 0.10 \\
$\mathrm{Si}_{3} \mathrm{~N}_{4}$ & & 0.10 & 0.12 \\
Durimide & & 0.10 & 0.15 \\
$\mathrm{SiO}_{2}$ & $\mathrm{GaAs}$ & 0.10 & 0.10 \\
$\mathrm{Si}_{3} \mathrm{~N}_{4}$ & & 0.10 & 0.12 \\
Durimide & & 0.10 & 0.12 \\
\hline
\end{tabular}

strongly absorbing in the visible spectral region but become transparent in the near infrared. Strong absorption will then become relevant again in the mid and far infrared. Therefore, most optical thickness determinations on semiconductors are carried out in the near infrared to the mid infrared with common reflectometers or FTIR. For highly doped semiconductors however, the transparency in this spectral region becomes weak what renders the optical thickness determination more difficult. The reason is that the doping introduces free 
charge carriers that change the optical properties of the material. The semiconductor becomes now absorptive. Then, the signal-to-noise ratio (SNR) of the used detector comes into play and determines which thickness can be detected maximum.

In the following we first show how the doping affects the refractive index $n$ and the absorption index or extinction coefficient $k$ of the semiconductor material for the example of silicon. Then, we derive a relation for the maximum evaluable thickness for absorbing layers. Finally, we summarize in a Table the maximum thickness in dependence upon the doping.

For calculation of the complex refractive index $n+i \cdot k$ of doped silicon we assume the dielectric function of doped silicon $\varepsilon^{*}$ as sum of the dielectric function of undoped silicon $\varepsilon$ plus a Drude susceptibility for the contribution of the dopants:

$\varepsilon_{\mathrm{Si}}^{*}(\omega)=\varepsilon_{\mathrm{Si}}(\omega)-\frac{\omega_{\mathrm{P}}^{2}}{\omega^{2}+\mathrm{i} \omega \gamma}$.

Note that in practice the silicon material gets doped already when producing the ingot. The reason is that pure undoped silicon has an intrinsic conductivity which is too low for application as electronic devices. The initial doping in the order of $10^{15} \mathrm{~cm}^{-3}$ is however too low to change the optical properties of silicon in the UV, visible, and near infrared spectral regions. Then, the material behaves optically similar to an undoped silicon.

The Drude susceptibility changes in dependence on the concentration $N$ of free charge carriers (electrons
But also the damping constant $\gamma$ in the Drude susceptibility is an important factor. This can be recognized best if we resolve Eq. (10) into real and imaginary part:

$$
\begin{aligned}
& \varepsilon_{1, \mathrm{Si}}^{*}(\omega)+i \cdot \varepsilon_{2, \mathrm{Si}}^{*}(\omega)=\varepsilon_{1, \mathrm{Si}}(\omega)-\frac{\omega_{P}^{2}}{\omega^{2}+\gamma^{2}} \\
& +\mathrm{i} \cdot\left(\varepsilon_{2, \mathrm{Si}}(\omega)+\frac{\gamma}{\omega} \frac{\omega_{\mathrm{P}}^{2}}{\omega^{2}+\gamma^{2}}\right) .
\end{aligned}
$$

While the real part of the dielectric function gets decreased by $\frac{\omega_{\mathrm{p}}^{2}}{\omega^{2}+\gamma^{2}}$, the imaginary part increases by this amount multiplied with $\gamma / \omega$. Hence, for a strong damping of the free charge carriers introduced by doping mainly the absorption in the wafer gets affected. The damping constant $\gamma$ can be retrieved from the conductivity $\sigma$ or the resistivity $\rho=1 / \sigma$ of the wafer using the relation

$\sigma=\varepsilon_{0} \frac{\omega_{\mathrm{P}}^{2}}{\gamma}$

presuming the conductivity or the resistivity has been measured for the whole wafer. Finally, using Maxwell's relation

$\mathrm{n}+\mathrm{i \kappa}=\sqrt{\varepsilon_{1}+\mathrm{i} \varepsilon_{2}}$

the complex refractive index is obtained from the changed dielectric function.

Absorption in the layer means that $K \neq 0$. Then, the magnitude of the wave reflected at the second interface layersubstrate (see Fig. 1) gets diminished and Eq. (1) changes to

$R(\lambda, d)=\frac{R_{01}(\lambda)+R_{12}(\lambda) \cdot E^{2}+2 \sqrt{R_{01}(\lambda) \cdot R_{12}(\lambda)} \cdot E \cdot \cos \left(\frac{4 \pi}{\lambda} n(\lambda) \cdot d\right)}{1+R_{01}(\lambda) \cdot R_{12}(\lambda) \cdot E^{2}+2 \sqrt{R_{01}(\lambda) \cdot R_{12}(\lambda)} \cdot E \cdot \cos \left(\frac{4 \pi}{\lambda} n(\lambda) \cdot d\right)}$

( $\mathrm{n}$-doped) or holes (p-doped)) as the plasma frequency $\omega_{\mathrm{p}}$ depends upon the concentration:

$\omega_{\mathrm{P}}^{2}=\frac{\mathrm{Ne}_{0}^{2}}{\mathrm{~m}_{\mathrm{eff}} \varepsilon_{0}}$.

In a parabolic band structure the effective mass $m_{\text {eff }}$ of the charge carriers is identical to the electron mass $m_{e}$ but in nonparabolic band structures $m_{e f f}$ may differ from $m_{e}$. For silicon the effective mass is $1.08 \cdot m_{e}$ for the electron and $0.56 \cdot m_{e}$ for the hole (derived from the density of states). For $m_{\text {eff }}=m_{e}$ we have approximately $\omega_{\mathrm{P}}^{2}=3.18261 \cdot 10^{9} \cdot \mathrm{N}\left[\mathrm{cm}^{-3}\right]$. with the abbrevation

$E=\exp \left(-\frac{4 \pi}{\lambda} \kappa \cdot d\right)$

The important result is that the oscillating cosine term is strongly decreased by absorption. This may cause problems for the detection of the oscillating term if its amplitude becomes smaller than the signal-to-noise ratio $S N R$ of the used detector. Assuming that the magnitude of the oscillating term is reduced to $1 / S N R$ we can derive the maximum measurable thickness $d_{m}$ to approximately

$\mathrm{d}_{\mathrm{m}} \approx(0.4-0.75) \cdot \frac{\lambda}{\kappa(\lambda)}$

for almost all materials and for SNR between 1000 and 5000 . 
Table 2 Maximum measurable thickness $d_{m}$ in microns for doped silicon with $N=10^{15} \mathrm{~cm}^{-3}$ to $N=10^{20} \mathrm{~cm}^{-3}$ and $\gamma=10^{15} \mathrm{~s}^{-1}$. All values are obtained for a signal-to-noise ratio $S N R=1000$

\begin{tabular}{lcrrrrr}
\hline$\lambda[\mathrm{nm}]$ & $\mathrm{N}\left[\mathrm{cm}^{-3}\right]$ & & & & \\
\cline { 2 - 6 } & $10^{15}$ & $10^{16}$ & $10^{17}$ & $10^{18}$ & $10^{19}$ & $10^{20}$ \\
\hline 1000 & 590 & 590 & 587 & 560 & 384 & 92.3 \\
1100 & 1028 & 1027 & 1017 & 927 & 491 & 85.8 \\
1200 & 1475 & 1473 & 1450 & 1251 & 527 & 77.4 \\
1300 & 1770 & 1766 & 1728 & 1425 & 516 & 69.7 \\
1400 & 2075 & 2069 & 2012 & 1578 & 499 & 63.4 \\
1500 & 2408 & 2399 & 2316 & 1719 & 480 & 58.3 \\
1600 & 2759 & 2746 & 2629 & 1843 & 461 & 54.0 \\
1700 & 3151 & 3134 & 2971 & 1957 & 443 & 50.4 \\
\hline
\end{tabular}

We calculated the thickness $d_{m}$ for a doped silicon wafer assuming a signal-to-noise ratio $S N R=1000$ and considered that also the reflectivity changes with the doping because the refractive index $n+\mathrm{i} \cdot k$ is affected by the doping. The results for $d_{m}$ in dependence on the concentration $N$ of free charge carriers and an assumed damping constant $\gamma=10^{15} \mathrm{~s}^{-1}$ are summarized in Table 2 .

For $N=10^{15}$ and $10^{16} \mathrm{~cm}^{-3}$ the effect of doping is negligible. These values are already obtained for undoped silicon from the intrinsic absorption of Si. The differences increase with $N$ and remarkable differences are obtained for $N=10^{18} \mathrm{~cm}^{-3}$. Up to this concentration it is however still possible to measure the thickness of uniformly doped silicon wafers of $1000 \mu \mathrm{m}$ thickness or less at wavelengths $\lambda>1100 \mathrm{~nm}$, presuming the signal-tonoise ratio is $S N R=1000$ or even higher. With a concentration of $N=10^{19} \mathrm{~cm}^{-3}$ the maximum thickness gets almost halved or even cut into thirds compared to $N=10^{15} \mathrm{~cm}^{-3}$ for wavelengths $\lambda<1400 \mathrm{~nm}$. For longer wavelengths the absorption caused by the free carriers reduces even more drastically the maximum measurable thickness. For $N=10^{20} \mathrm{~cm}^{-3}$ the maximum thickness is finally less than $100 \mu \mathrm{m}$ at all wavelengths.

\section{Summary}

The use of fast Fourier transform (FFT) is a fast method to determine the thickness of a transparent layer on a substrate or the thickness of a substrate from the measured reflectance spectrum. Beyond the well-known problems with discrete Fourier transforms in signal processing, its application in layer thickness determination is however rendered more difficult by problems that are specific for this application. The main problems are caused by the non-equidistant wavelength steps in the wavelength interval of the spectrometer and by the dispersion, i.e. the wavelength dependence of the refractive index. The non-equidistant wavelengths $\lambda$ in the spectrometer evoke Moiré effects in the measured reflectance of a layer. These
Moiré effects cannot be eliminated by transformation of the reflectance spectrum to wavenumbers $1 / \lambda$. In the Fourier transform they generate a band of frequencies higher than the Nyquist frequencies and appear as band of alias frequencies in the power spectral distribution. As long as this band does not appear at frequencies lower than the layer thickness frequency a sophisticated peak search algorithm will find the layer thickness frequency. Yet, when shifting to lower frequencies the modulus of these alias frequencies strongly increases which strongly disturbs the peak search algorithm. In this way they restrict the layer thickness determination on a certain size still far below the Nyquist frequency. A helpful method is to investigate a modified PSD, namely the PSD multiplied with the square of the pixel number in the PSD. Then, a thickness close to the Nyquist frequency can clearly be determined if the smallest evaluable thickness given by the used spectrometer is larger than $4 \mu \mathrm{m}$.

The dispersion of the refractive index $n$ of the layer is problematic manifold. First of all, from a mathematical view it prevents the application of a Fourier transform since "time" and "frequency" are no further independent. The use of a constant value is only helpful if this value amounts to a certain value $n_{\text {eff }}$ which can be calculated for each material in dependence on the used spectral range. Although this is a reasonable way to improve the layer thickness determination with FFT it does not prevent the Moire pattern in the reflectance spectrum. In contrast, as it is an intrinsic material property it cannot be neglected when measuring the reflectance. That means that even if we would arrive in equidistant wavelength steps in the measurement, the nonlinear dispersion of $n$ would make the phase of the oscillating term non-equidistant.

Improvements of the FFT results are possible but need an opportunity to calculate reflectance spectra for a layer on a substrate. Then, a first improvement is a simple grid search around the thickness determined by FFT analysis. Further improvement is obtained using the result of this grid search as initial value for a nonlinear regression. These 
methods are time-consuming but increase the accuracy of the FFT analysis by minimum a factor of 500-1000.

Finally, we showed that it is possible to determine layer thickness with FFT on structured samples if the contribution of the layer reflectance to the total reflectance measured on a detection area on the sample is larger than $15 \%$. Second, we also demonstrated for the example of highly doped silicon the influence of absorption in the layer on the maximum evaluable thickness. This maximum thickness not only depends upon the absorption but also upon the signal-to-noise ratio of the used spectrometer because the absorption strongly decreases the magnitude of the oscillating term that contains the information on the thickness.

\section{Compliance with ethical standards}

Conflict of interest The author declares that they have no conflict of interest.

\section{References}

1. Quinten M (2012) A practical guide to optical metrology for thin films. Wiley, Weinheim

2. Palik ED (Ed.) (1985) Handbook of optical constants of solids I, Academic Press, San Diego; (1991) Handbook of optical constants of solids II, Academic Press, San Diego; (1998) Handbook of optical constants of solids III, Academic Press, San Diego
3. Humlicek J, Carriga M, Alonso MI, Cardona M (1989) Optical spectra of $\mathrm{Si}_{\mathrm{x}} \mathrm{Ge}_{(1-\mathrm{x})}$ alloys. J Appl Phys 65:2827-2832

4. Green MA, Keevers MJ (2007) Optical properties of intrinsic silicon at 300 K. Progr Photovolt Res Appl 3:189-192

5. Houta F, Quinten M (2015) Layer thickness determination-the influence of the aperture of a measuring head. Opt Photon 10(4):54-56

6. Quinten M, Houta F, Fries T (2015) Problems in thin film thickness measurement resolved: improvements of the fast fourier transform analysis and consideration of the numerical aperture of microscope headers and collimators. In: Proceedings of SPIE 9526 modeling aspects in optical metrology V, 95260R

7. Bass M (ed) (1994) Handbook of optics, Vol. 2: Devices, measurements, and properties, 2nd edn. McGraw-Hill Professional, New York

8. Taylor \& Francis Group (2014-2015) CRC handbook of chemistry and physics, CRC Press, Boca Raton, 95th edition

9. Adacho $S$ (1999) Optical constants of crystalline and amorphous semiconductors: numerical data and graphical information. Kluwer Academic Publications, Norwell

10. Ward $L$ (1994) The optical constants of bulk materials and films, 2nd edn. Institute of Physics Publishing, Bristol

11. Levenberg K (1944) A method for the solution of certain problems in least squares. Quart Appl Math 2:164-168

12. Marquardt $D$ (1963) An algorithm for least-squares estimation of nonlinear parameters. SIAM J Appl Math 11:431-441

13. Nelder JA, Mead R (1965) A simplex method for function minimization. Comp J 7:308-313

14. Li Ya-Ping, Lee Cheng-Chung (2007) Simulation of intensity of a patterned thin-film structure. Appl Opt 46(12):2244-2247

Publisher's Note Springer Nature remains neutral with regard to jurisdictional claims in published maps and institutional affiliations. 